\title{
Viscosity and aggregation structure of nanocolloidal dispersions
}

\author{
WANG Tao ${ }^{1,2}$, NI MingJiang ${ }^{1}$, LUO ZhongYang ${ }^{1 *}$, SHOU ChunHui $^{1} \& \mathrm{CEN} \mathrm{KeFa}^{1}$ \\ ${ }^{1}$ State Key Laboratory of Clean Energy Utilization, Zhejiang University, Hangzhou 310027, China; \\ ${ }^{2}$ Earth Institute and Department of Earth and Environmental Engineering, Columbia University, New York, NY 10027, USA
}

Received November 18, 2011; accepted March 1, 2012

\begin{abstract}
In this work, the effects of nanoparticle size, particle volume fraction and $\mathrm{pH}$ on the viscosity of silicon dioxide nanocolloidal dispersions are investigated. Both size and $\mathrm{pH}$ are found to significantly affect nanocolloid viscosity. Two models are used to study the effect of aggregate structure on the viscosity of the nanocolloidal dispersion. The fractal concept is introduced to describe the irregular and dynamic aggregate structure. The structure of aggregates, which is considered to play an important role in viscosity, is affected by both intermolecular and electrostatic forces. The particle interaction is primarily affected by particle distance and becomes stronger with decreasing particle size and increasing volume fraction. The aggregate structure is also affected by the $\mathrm{pH}$ of the solution. Studying the relationship between $\mathrm{pH}$ and zeta-potential shows that with the neutralization of charges on the particle surface and decreasing electrical repulsion force, the particle interaction becomes dominated by attractive forces and the aggregates form a more compact structure.
\end{abstract}

nanocolloid, viscosity, aggregation, fractal model

Citation: Wang T, Ni M J, Luo Z Y, et al. Viscosity and aggregation structure of nanocolloidal dispersions. Chin Sci Bull, 2012, 57: 3644-3651, doi: $10.1007 / \mathrm{s} 11434-012-5150-\mathrm{y}$

In recent years, nanocolloidal dispersions, which are heterogeneous mixtures consisting of very small particles with sizes typically in the order of 1-1000 nm, have attracted much attention for applications related to cooling [1], nanolubricants [2], drug delivery and diagnosis [3]. In the application of nanocolloidal dispersions, the viscosity, which is related to the required pumping power and diffusion properties, plays an important role in delivery systems. The rheological behavior of nanoparticle-fluid mixtures have been investigated experimentally [4-7]. Newtonian behavior has been observed for very dilute suspensions $[4,7]$ and a considerable amount of effort has been devoted to determining the relationship between the viscosity and volume fraction of suspensions. $\mathrm{Yu}$ et al. [8] observed shear-thinning behavior when the volume concentration was higher than 0.03. An interesting phenomenon is that most of the viscosities are under-predicted by the traditional Einstein viscosity model $[9,10]$. This discrepancy could be

\footnotetext{
*Corresponding author (email: zyluo@zju.edu.cn)
}

caused by aggregation of the nanoparticles and an underestimated effective volume fraction of clusters [10,11]. Rubio-Hernandez et al. [6] also noted that the intrinsic viscosity will be affected by the shape of aggregates, which could be changed by the $\mathrm{pH}$ and shear rate of a colloidal system.

The formation of aggregates can greatly change the transfer of heat and stress in a system because of their porous structure and large effective volume fraction. Particularly for nanocolloidal dispersions, the distance between aggregates can be in the order of several nanometers. The aggregation of nanoparticles can simply be characterized as ellipsoidal with a certain effective size and axial ratio. The size and shape of aggregates can be determined from dynamic scattering light measurements and intrinsic viscosity data, respectively [6]. Aggregation is a dynamic process and the structure changes continuously because of Brownian motion. The dynamic structure of aggregates is too irregular to be easily described by traditional Euclidean geometry. In recent years, the fractal concept has been introduced to understand aggregation phenomena in colloids. Rooij et al. [12] 
used the fractal model to analyze aggregation in a polymer dispersion and studied the shear viscosity. Prasher et al. [10,11] and Jiang et al. [13] studied the kinetic process of nanoparticle aggregation, in which fractal models were used to describe the structures of nanoparticle aggregates and were combined with the effective medium theory to predict their physical properties. For the kinetic model study, the formation process and structure of an aggregate are related to particle-particle interactions. Aggregates formed in the diffusion-limited regime, which indicates a weak repulsive barrier and less compact aggregate structure, are considered to have a fractal dimension of about 1.8 [11].

From the experimental work reviewed above, aggregation plays an important role in determining the physical properties of nanocolloidal dispersions, and the combination of fractal and effective medium theories can be used to predict the effects of aggregation. However, a common problem in the above studies is that the fractal dimension, which is one of the basic parameters used to describe fractals, was set as a fixed value for all conditions. In reality, the structure of aggregates can change with particle size, concentration or $\mathrm{pH}$ because of changed particle interactions. Determination of aggregate structure can not only reveal the interactions between nanoparticles but also help to comprehend the anomalous physical properties of nanocolloidal dispersions. The structure of nanoparticle aggregates can be characterized directly by experimental methods. Wang et al. [14] measured the fractal dimension of aggregates by direct topological analysis following rapid freezing in liquid helium. However, thousands of samples have to be analyzed to achieve statistical significance. Moreover, it is difficult to describe an aggregate structure in three dimensions by topological analysis. Waite et al. [15] used the static light scattering technique to determine the fractal structure of an alumina nanoparticle solution. Their experimental results fit the theoretical model well. However, this optical technique requires a very dilute sample, with a volume fraction of less than $0.5 \%$ [15].

In this paper, the viscosities of $\mathrm{SiO}_{2}$ nanoparticle dispersions containing different particle sizes and volume concentrations were measured. The prolate ellipsoid and fractal model were used to study the effects of aggregation on viscosity. The structure of the aggregates was investigated to reveal the physics behind the size and concentration effects of nanocolloidal dispersions. The relationship between $\mathrm{pH}$, zeta $(\zeta)$ potential and viscosity were also measured to study the effect of electrostatic interactions on aggregate structure and viscosity.

\section{Experimental methods and viscosity model}

\subsection{Material preparation}

A microfluidic high shear dispersion method was used to prepare the $\mathrm{SiO}_{2}$ dispersions. Spherical $\mathrm{SiO}_{2}$ nanoparticles (white powder) with diameters of 7, 12, 16, 20 and $40 \mathrm{~nm}$ were supplied by Degussa (Evonik, Germany). The powders were dispersed in deionized (DI) water and deagglomerated with a microfluidic high shear fluid processor (Microfluidizer M-110S, Microfluidics Corporation, USA). The processor contained an air-powered intensifier pump designed to supply the desired pressure at a constant rate to the dispersion stream. As the pump travels through its pressure stroke, it drives the dispersion at constant pressure (in the range of 200 to $1600 \mathrm{~atm}$ ) through microchannels with fixed geometry within the interaction chamber. As a result, the dispersion stream accelerates to high velocity, creating a shear rate within the stream that is in the order of $10^{7} \mathrm{~s}^{-1}$. SEM (JEM 200CX) images of the dispersed nanoparticles are shown in Figure 1. The particles were spherical and uniform after deagglomeration at high shear rate.

\subsection{Property characterization}

The size of the aggregates and $\zeta$-potentials of the dispersions were determined by dynamic light scattering using a Malvern Zetasizer Nano S90. The rheological properties of the nanocolloidal dispersions were measured by a rheometer (Haake Instrument). The viscosities of the suspensions were determined by Ubbelohde-type capillary viscometers. The

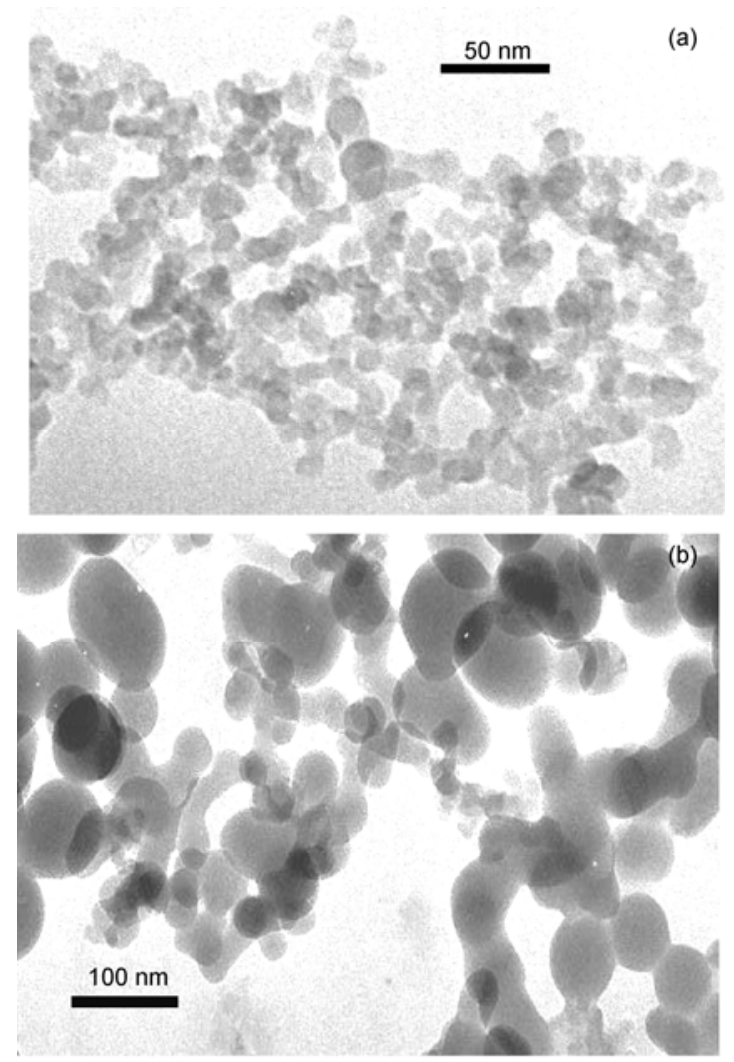

Figure 1 SEM images of dispersed $\mathrm{SiO}_{2}$ nanoparticles with a diameter of (a) $7 \mathrm{~nm}$, and (b) $40 \mathrm{~nm}$. 
capillary viscometers were calibrated using DI water. Observation experiments showed that no sedimentation occurred in the first three months following colloid preparation. Even so, all samples were prepared and tested within $48 \mathrm{~h}$ at $25^{\circ} \mathrm{C}$. Repeat experiments showed that the capillary viscometer had an experimental error of about $3 \%$.

\subsection{Viscosity model}

(i) Prolate ellipsoid model. For a nanocolloidal dispersion, a power series of volume fraction, which takes into account particle-particle interactions for several orders, was used to predict the viscosity [6]:

$$
\eta=\eta_{0}\left(1+[\eta] \phi+K[\eta]^{2} \phi^{2}+O\left(\phi^{3}\right)+\cdots\right),
$$

where $\eta_{0},[\eta], K$ and $\phi$ are the viscosity of the base fluid, intrinsic viscosity, Huggins coefficient and the particle volume fraction, respectively. $[\eta]$ reflects the capability of particles/aggregates to increase the viscosity of a colloid, and is strongly related to the shape of aggregates though the Simha expression [16]. Sun et al. [17] obtained a simple equation relating $[\eta]$ to the axial ratio of a prolate ellipsoid in the range of $1<a / b<100$ by a Newtonian approximation to the results of Simha:

$$
[\eta]=0.057(a / b)^{2}+0.61 a / b+1.83
$$

where $a$ and $b$ are the longer and shorter semidiameters of an ellipsoid, respectively. The effect of aggregate shape on viscosity is obvious, and the intrinsic viscosity determined using eq.(2) are plotted in Figure 2. $[\eta]$ increases quickly from 2.5 to 630 as the aggregate turns from a sphere to an ellipsoid with an axial ratio of 100. Carbon nanotubes (CNTs), for example, are considered to have great potential for enhancing thermal conductivity because of their large axial ratio and phonon mean free path [18]. Assuming CNTs with an axial ratio of 100 are well dispersed in solution, the increase in viscosity would be six times higher than that of the base fluid at a volume fraction of $1 \%$. This very large increase in viscosity may weaken the improvement of

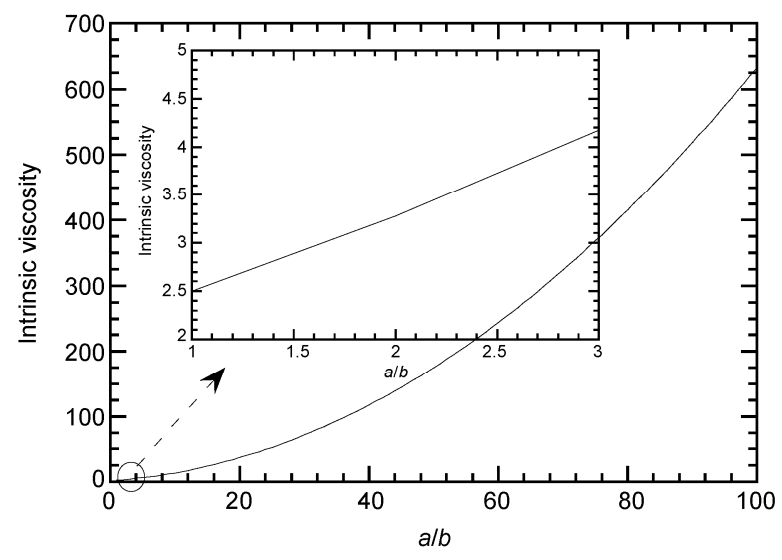

Figure 2 The effect of particle/cluster shape on intrinsic viscosity. heat transfer in the nanocolloidal dispersion during application.

(ii) Fractal model. The Fractal model uses the concept of self-similarity to describe aggregate structure. The parameter of fractal dimension is a statistical quantity and used to indicate how completely a fractal appears to fill space as the scale decreases. The number of particles contained inside a sphere of radius $\lambda$ inside an aggregate (the origin $\lambda=0$ is supposed to be in the center of gyration of the aggregate) is given by [12]

$$
N=(\lambda / r)^{d_{f}},
$$

where $r$ is the particle radius and $d_{f}$ is the fractal dimension. Then, the particle volume fraction in an aggregate of gyration radius $R_{g}$ can be easily calculated as

$$
\phi_{p a}=\left(R_{g} / r\right)^{d_{f}-3} .
$$

According to Krieger's formula, the viscosity of an aggregated colloid is given as [19]

$$
\eta=\eta_{0}\left(1-\phi_{a} / \phi_{m}\right)^{-2.5 \phi_{m}}
$$

where, $\phi_{m}$ is the volume fraction of densely packed spherical particles, and is chosen to be 0.63 , which is the value for random close packing [12]. $\phi_{a}$ is the volume fraction of aggregates and is equal to $\phi / \phi_{p a}$. The Krieger model considers the effect of aggregation and can be applied to colloidal dispersions with a high volume fraction. The aggregate in this formula is assumed to be spherical. For dilute and well dispersed colloids, the formula can be reduced to the Einstein equation [20], in which $[\eta]$ is 2.5 . Combining eqs. (4) and (5), one can obtain the fractal dimension of an aggregate as

$$
d_{f}=3-A(B-\log \phi),
$$

where, the coefficients $A$ and $B$ are $1 / \log \left(R_{g} / r\right)$ and $\log \left[\phi_{m}\left(1-\eta_{\text {rel }}^{-1 /\left(2.5 \phi_{m}\right)}\right)\right]$, respectively. $\eta_{\text {rel }}$ is the relative viscosity which is equal to $\eta / \eta_{0} . B$ can be obtained directly from the measured increase in viscosity. The gyration radius can be simply estimated by an empirical relationship of $R_{g}=R_{h} / 0.8$ [12,21], where $R_{h}$ is the hydrodynamic radius of an aggregate and can be measured by dynamic light scattering.

\section{Results and discussion}

The rheological properties of $\mathrm{SiO}_{2}$ nanocolloidal dispersions containing particles with a diameter of $7 \mathrm{~nm}$ were studied, and are shown in Figure 3. The shear stress is plotted against the shear rate for volume fractions of $1 \%, 2 \%$ and $4 \%$. The shear stress depends linearly on the shear rate and the viscosity is constant, indicating the nanocolloidal dispersions exhibit Newtonian behavior under the conditions 


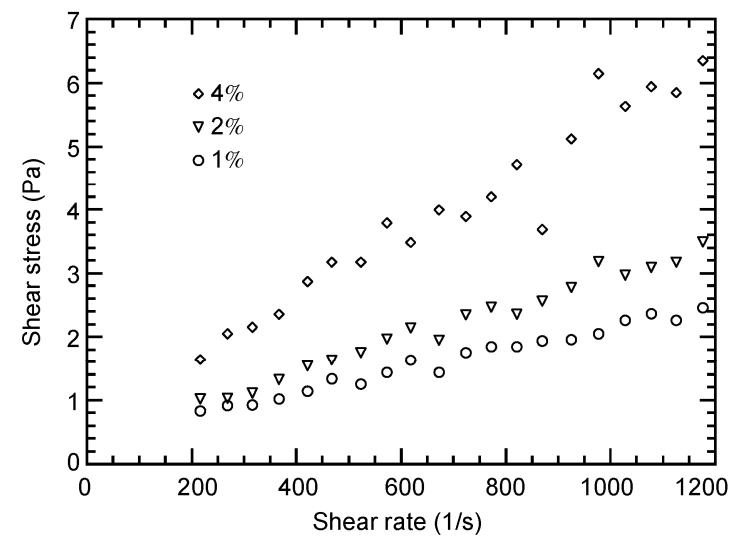

Figure 3 Rheological behavior of nanocolloidal dispersions of $\mathrm{SiO}_{2}$ nanoparticles with a diameter of $7 \mathrm{~nm}$.

used in this study. Newtonian behavior of nanocolloidal dispersions with low volume fraction is consistent with previous research $[4,7,8]$.

\subsection{Intrinsic viscosity and size effect}

The viscosity of nanocolloidal dispersions with different sizes and volume fractions were measured. According to eq.(1), $[\eta]$ can be determined from the intercept of the straight line of $\left(\eta / \eta_{0}-1\right) / \phi$ as a function of $\phi$. The term $\left(\eta / \eta_{0}-1\right) / \phi$ is known as the reduced viscosity. Figure 4 shows the reduced viscosities for nanocolloidal dispersions containing different particle sizes. Viscosity increases with decreasing particle size for the same volume fraction. This size effect could be caused by the stronger interaction between smaller particles compared with that between larger ones. For a dilute dispersion without interparticle forces, the viscosity will be governed by classic Einstein behavior and no size effect is expected. In this study, the slope of the reduced viscosity curve, or the Huggins coefficient $K$ in eq.(1), increases with decreasing particle size. This indicates that even for dilute nanocolloidal dispersions, the effect of particle interaction on viscosity should be considered, especially for smaller particles. The nanoparticles in dispersions can easily interact with each other because of the relatively short distance between them. As calculated by Keblinski et al. [22], the average surface-to-surface distance between 10 $\mathrm{nm}$ particles is only $5 \mathrm{~nm}$ if the volume fraction is $5 \%$. Moreover, the strong Brownian motion of small particles can enhance their particle-particle interactions. $[\eta]$ determined for the dispersions varies from 20 to 60 with decreasing particle size. It is known that, without considering the aggregation of particles, $[\eta]$ of dilute suspensions of spherical particles is 2.5 [20]. The $\mathrm{SiO}_{2}$ particles in this experiment are spherical, but the aggregate structure also has to be considered. There are two forces that dominate the interaction between particles: the intermolecular force (also known as the van der Waals force), and the electrostatic force that is caused by the electrical double layer on the surface of the particle. The electrostatic force is repulsive because of the charges on the surface of the same particles are same. The role of these two forces will vary with different particle size and volume fraction, affecting the degree and structure of the aggregate. For example, when the electrostatic force is larger than the intermolecular force, the aggregates will tend to form a chain because of the repulsive interaction between particles. A large repulsive force can even cause the particles to deagglomerate, which is the mechanism of some nanoparticle dispersants. According to the prolate ellipsoid model, the structure of an aggregate is related to $[\eta]$ by eq. $(2)$. $[\eta]$ of the suspensions and the axial ratios of the ellipsoidal aggregates with different particle sizes are listed in Table 1. As the particle diameter decreases, the axial ratio increases, which means that the aggregate tends to be a more 'prolate' ellipsoid.

The relative viscosities for nanocolloidal dispersions containing $\mathrm{SiO}_{2}$ particles with a diameter of $7 \mathrm{~nm}$ are presented in Figure 5. Previous models for Newtonian fluids with low volume fraction were also included. The experimental data in this research is slightly higher than that

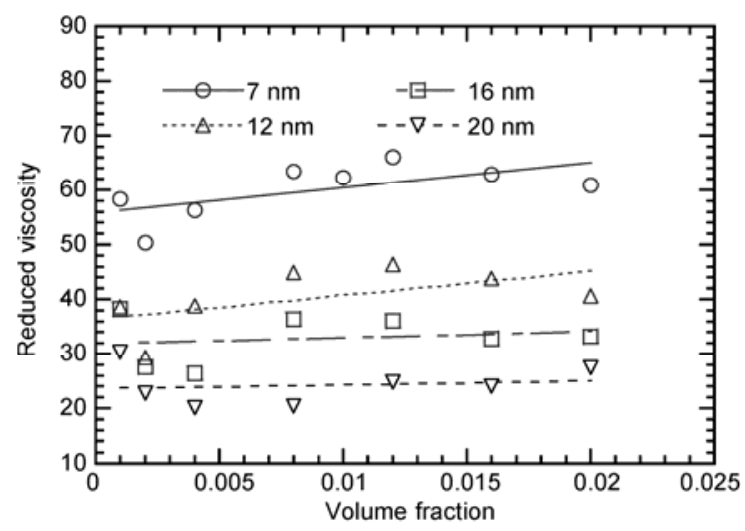

Figure 4 Intrinsic viscosities for nanocolloids containing different particle sizes. Dots represent experimental results. Lines represent linear fitting results.

Table 1 Particle size, measured intrinsic viscosity and effective axial ratio of clusters in different nanocolloidal dispersions

\begin{tabular}{|c|c|c|c|c|}
\hline Particle diameter (nm) & Hydrodynamic radius (nm) & Gyration radius $(\mathrm{nm})$ & {$[\eta]$} & $a / b$ \\
\hline 7 & 114 & 143 & 55.9 & 25.9 \\
\hline 12 & 131 & 164 & 36.5 & 19.9 \\
\hline 16 & 144 & 180 & 31.9 & 18.2 \\
\hline 20 & 165 & 206 & 23.7 & 15.0 \\
\hline 40 & 196 & 245 & 18.3 & 12.5 \\
\hline
\end{tabular}




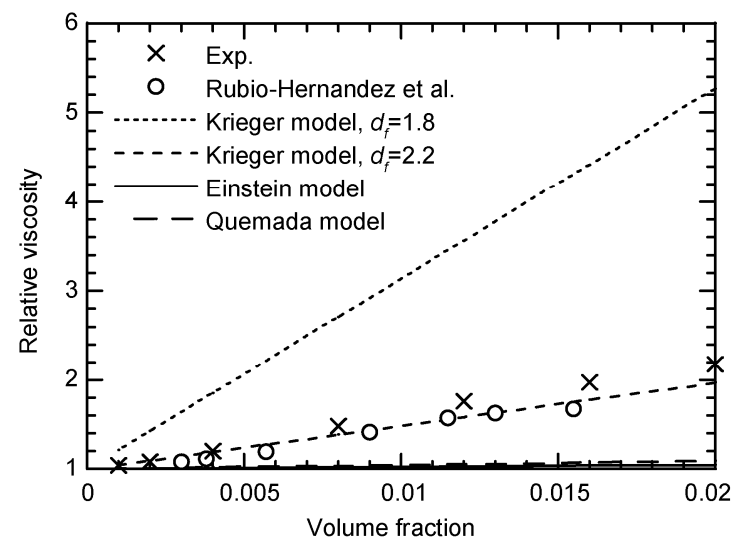

Figure 5 Relative viscosities for nanocolloidal dispersions containing $\mathrm{SiO}_{2}$ particles with a diameter of $7 \mathrm{~nm}$. The crosses are experimental results measured in this study.

measured by Rubio-Hernandez et al. [6]. This difference may caused by the different $\mathrm{pH}$ of the two dispersions, which were 5.3 and 6.1 for this research and that of $\mathrm{Ru}-$ bio-Hernandez, respectively. The effect of $\mathrm{pH}$ on viscosity will be discussed in detail later. The viscosity data were under-predicted by models from classical effective medium theory, like the Einstein and Quemada models [23]. Moreover, the classical models did not predict the strong size effect illustrated in Figure 4. It is possible that the failure of classical models to accurately describe viscosity is related to the aggregation of nanoparticles. The effect of aggregation on viscosity is reflected in the Krieger model with $d_{f}$. Figure 5 shows that the viscosities depend strongly on $d_{f}$. However, the fractal structure is affected by many factors including particle size, volume fraction and $\mathrm{pH}$ of a dispersion. It is unreasonable to assume a fixed value for $d_{f}$ under different conditions.

\subsection{Effect of volume fraction on aggregate structure}

The prolate ellipsoid model gives us an idea how the particle size affects particle interactions and thus the viscosity. It is known that, as well as particle size, interparticle forces are also affected by other factors including particle volume fraction, $\mathrm{pH}$ and external field. Any of these factors can change the structure of an aggregate. The prolate ellipsoid model is based on the concept of $[\eta]$ and does not reflect the change in aggregate structure with particle volume fraction. $d_{f}$, as shown in eq.(6), is directly related to the aggregate volume fraction and viscosity of a colloid. From eq.(3), it is known that more particles will be contained inside a sphere of the same radius within an aggregate with a larger $d_{f}$. In view of this, $d_{f}$ gives an indication of how completely a fractal appears to fill space. Figure 6 shows the dependence of $d_{f}$ on particle size and volume fraction. The sizes of the aggregates were measured by dynamic light scattering, and the results are listed in Table 1.

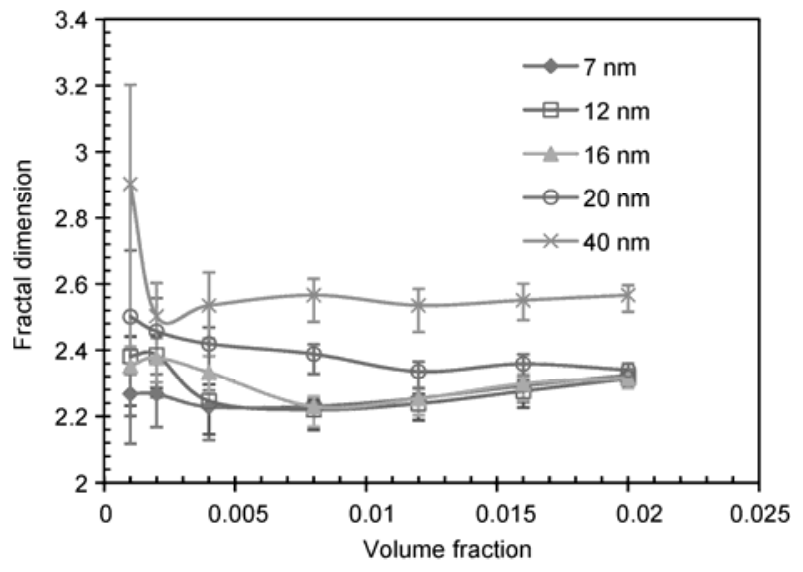

Figure 6 Fractal dimension of aggregates containing nanoparticles of different sizes.

It is interesting to find that the fractal model reveals a strong relationship between $d_{f}$ and particle size. $d_{f}$ increases with increasing particle size, especially at lower volume fractions $(<0.005)$. In this situation, as shown using the prolate ellipsoid model, the aggregate approaches to a chain structure because of a stronger repulsive force between smaller particles. This means that an aggregate containing smaller particles will form a less compact structure and may possess a smaller $d_{f}$. For nanoparticles with a diameter of 40 $\mathrm{nm}$, the abrupt increase of $d_{f}$ when the volume fraction is less than $0.2 \%$ could also arise from the error in the viscosity measurement. In eq.(6), $B$ has a logarithmic relationship with the relative viscosity. At lower particle volume fractions, the relative viscosity is close to 1 and $1-\eta_{\mathrm{rel}}^{-1 /\left(2.5 \phi_{m}\right)}$ would approach 0 . A viscosity error of $\pm 3 \%$ could then contribute to an error of $\pm 30 \%$ for $d_{f}$ at lower volume fractions. The errors of $d_{f}$ induced by the error in viscosity measurement are presented as error bars in Figure 6. At the same time, $d_{f}$ is also dependent on volume fraction. Figure 6 shows that $d_{f}$ decreases with increasing volume fraction and then reaches a stable level. It is known that the interaction between particles is related to distance. Two particles tend to attract each other and form an aggregate when they are separated. However, repulsive forces will dominate the interaction if the two particles are too close, such as several nanometers apart. Under very dilute conditions, most of the nanoparticles only interact with each other within the aggregate. As the particle volume fraction increases, the reduced distance between aggregates will greatly increase the chance of particle collision and interaction between different aggregates. When two aggregates are close enough, the particles at the surface of an aggregate will be attracted by the other aggregate until a new equilibrium state is formed. Figure 7 shows that aggregates with less compact structures and larger effective volume fractions will be formed as the distance between aggregates decreases.

It is important to note that with increased particle volume 
fraction, the average distance between aggregates can be in the order of several particle diameters. Then, aggregates may merge into bigger ones because of the strong interactions between them. In this research, the particle volume fractions were controlled below $2 \%$ and no reagglomeration with increased volume fraction is assumed. To show the effect of particle volume fraction on aggregate interaction, the effective volume fraction and average distance between aggregates are plotted in Figure 8. It is surprising to find that the volume fraction of aggregate for the dispersion containing particles with a diameter of $7 \mathrm{~nm}$ can reach about $25 \%$ when the particle volume fraction is $2 \%$. Moreover, the surface distance decreases quickly with increasing volume fraction, which provides evidence for the stronger interaction between aggregates as the volume fraction increases. For a dispersion containing particles with a diameter of $7 \mathrm{~nm}$, the surface distance between aggregates is only tens of nanometers when the volume fraction is larger than $1 \%$, which will greatly increase the chance of collision between aggregates. The nonlinear curves of aggregate volume fraction for dispersions containing particles with diameters of 7 and $12 \mathrm{~nm}$ also indicate that reagglomeration occurs. The viscosity model for nanocolloidal dispersion should be carefully built when considering that reagglomeration can occur at high volume fraction.
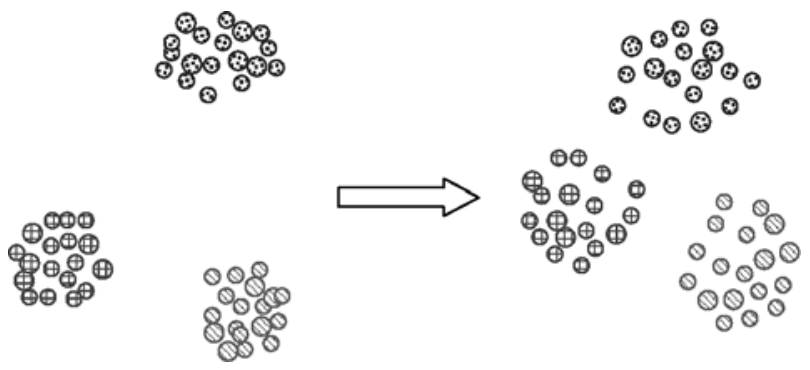

Figure 7 Change in the structure of aggregates with increasing volume fraction.

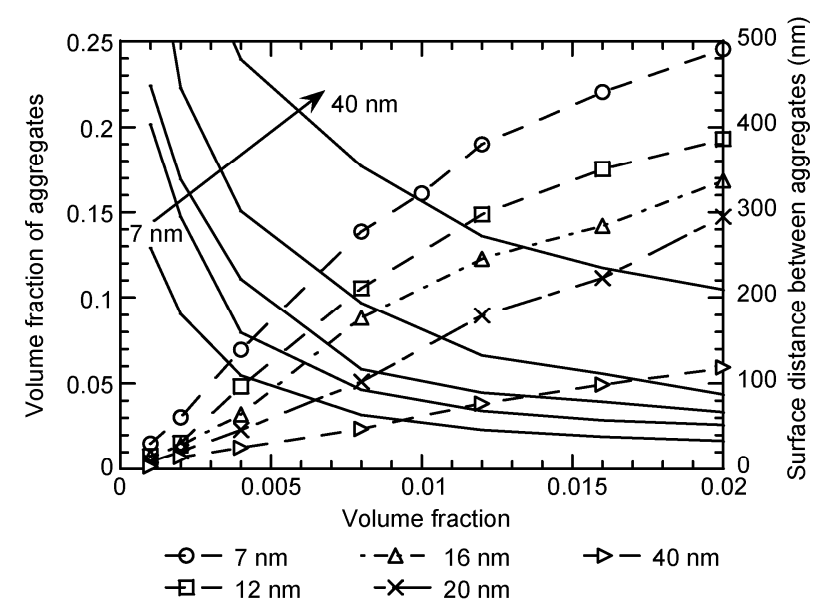

Figure 8 Volume fraction and average surface distance between aggregates. Solid lines are the surface distance. Broken lines are the volume fraction.

\subsection{Effect of $\mathrm{pH}$ on aggregate structure}

To further study the effect of particle interaction on aggregation structure, the viscosity of the colloidal system was measured at different $\mathrm{pH}$. The $\zeta$-potentials of the colloids were measured to characterize the electronic interactions. This parameter indicates the degree of repulsion between particles and can be related to the stability of colloidal dispersions. $\zeta$-potentials were determined by measuring the electrophoretic mobility of the colloids.

Figure 9 shows the effect of $\mathrm{pH}$ on the viscosity of the colloids. The initial $\mathrm{pH}$ of the colloid was about 5.3 and was adjusted readily by addition of dilute $\mathrm{HCl}$ solution. The slightly acidic colloid in the initial state may be caused by acid species being adsorbed on the particle surface during preparation. As can be seen, the relative viscosity decreases quickly with as the $\mathrm{pH}$ of the dispersions is decreased, and approaches stability when the $\mathrm{pH}$ is less than 5. At the same time, $d_{f}$ of the aggregate increases quickly and then becomes stable as the $\mathrm{pH}$ decreases. The effect of $\mathrm{pH}$ on $d_{f}$ indicates that the structure of the aggregate could be changed by the $\mathrm{pH}$. It is known that there is an electrical double layer at the interface between a particle and aqueous solution. The first layer, which is also called the stern or stationary layer, is comprised of ions adsorbed directly onto the particle surface. The second layer is composed of ions attracted to the surface charge via Coulombic forces, and electrically screens the stationary layer. This second layer is loosely associated with the particle, because it is made of free ions that can move in the fluid under the influence of electric attraction and thermal motion rather than being firmly anchored. As a result, it is often called the diffuse layer. When the particle moves, the diffuse layer, or at least part of it, can move as part of the particle. The plane that separates the dispersion medium from the fluid layer that remains attached to the particle surface is called the slipping plane. The $\zeta$-potential is the potential difference between the dispersion medium and slipping plane. In this study, the sign of the measured $\zeta$-potential of the colloids is negative, which means that the

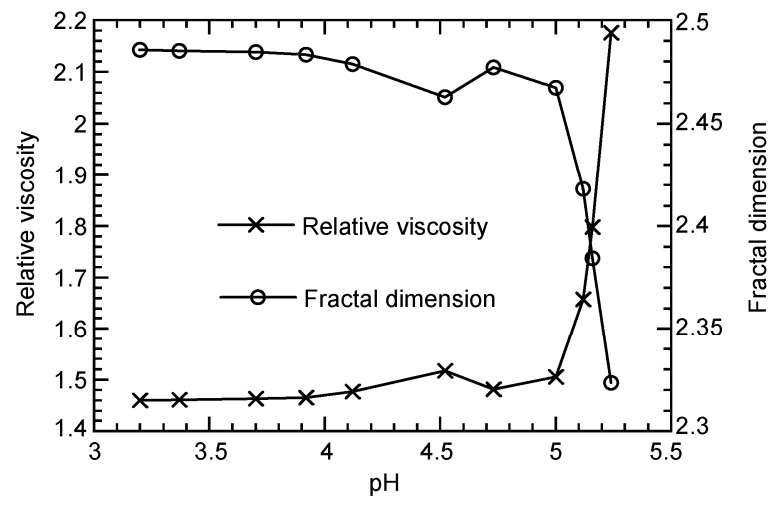

Figure 9 The effect of $\mathrm{pH}$ on the relative viscosity and fractal dimension of a colloidal dispersion. The dispersion had a volume fraction of $2 \%$ and contained particles with a diameter of $7 \mathrm{~nm}$. 
charge at the particle surface is negative. As the $\mathrm{pH}$ of the nanocolloidal system is decreased, the negative surface charge would be readily neutralized. Thus, the repulsive electrostatic interaction will be weakened as the thickness of the double layer decreases.

The effect of $\mathrm{pH}$ on the $\zeta$-potential was measured, and the results are illustrated in Figure 10. When the $\mathrm{pH}$ of the colloid was adjusted from 5.3 to 3.2 , the $\zeta$-potential decreased from about -17 to $-1 \mathrm{mV}$. As discussed above, the decreased absolute value of $\zeta$-potential is caused by the decreased electrical repulsion force due to charge neutralization. As a result, the aggregate forms a more compact structure, as indicated by the change in $d_{f}$ shown in Figure 9. The particle volume fractions in a cluster were also calculated and the results were plotted against the $\mathrm{pH}$ of the colloid system (Figure 10). As the colloid becomes more acidic, the absolute value of the $\zeta$-potential decreases quickly from about $17 \mathrm{mV}$ to near zero and the particle volume fraction in the aggregate increases from about $8 \%$ to $15 \%$. Another phenomenon is that when the $\mathrm{pH}$ is below 4.0 and the $\zeta$-potential is less than $5 \mathrm{mV}$, the particle volume fraction in the aggregate tends to be stable. This indicates that van der Waals forces begin to dominate the particle interactions in this region, so the aggregates tend to form a stable structure.

Compared with the classic Einstein equation, the viscosity of the colloid is still underestimated because the aggregate is assumed to possess a spherical structure in this study. The relative viscosity predicted by the Einstein equation is 1.05 for a nanocolloidal dispersion with a volume fraction of $2 \%$. Figure 9 shows that the experimental value is larger than 1.4. This underestimation could be because of the large volume of the aggregate, which changes from $13 \%$ to $24 \%$ as the $\mathrm{pH}$ is adjusted. Our findings further prove that aggregation has a significant effect on colloid viscosity.

\section{Conclusions}

In summary, the viscosities of $\mathrm{SiO}_{2}$ nanocolloidal dispersions containing different particle sizes and volume frac-

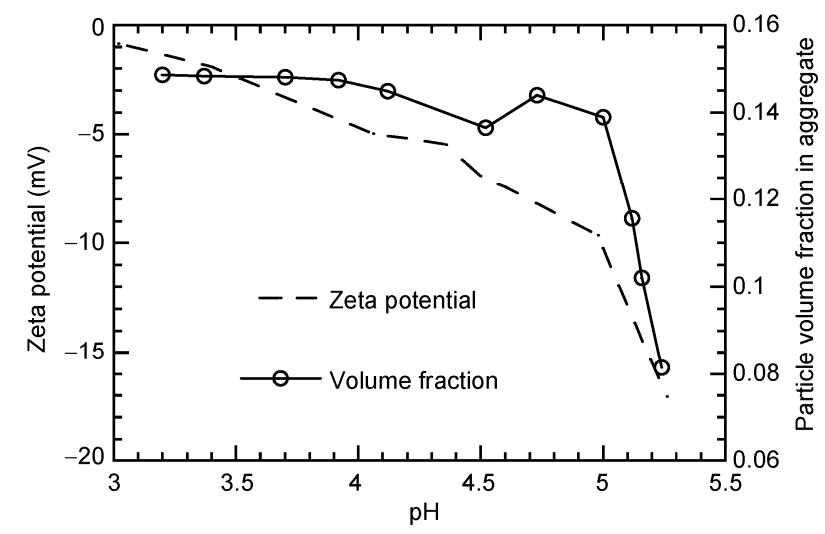

Figure 10 Relationship between Zeta potential and aggregate structure. tions were measured. A significant effect of size on relative and intrinsic viscosity was observed experimentally. Two models were used to study the effect of aggregation structure on the viscosity of nanocolloidal dispersions. The prolate ellipsoid model shows that as the particle diameter decreases, the axial ratio increases, which means the aggregate tends to form a more 'prolate' ellipsoid. The fractal model uses the concept of self-similarity to describe aggregation structure. It can reveal more information than the prolate ellipsoid model by considering the irregular and dynamic properties of aggregates. The structure of aggregates, which is considered to play an important role in viscosity, is affected by both intermolecular and electrostatic forces. With increasing particle concentration and decreasing average distance between aggregates, the particles at the surface of different aggregates attract each other and a less compact aggregation structure is formed. The measured relationships between $\mathrm{pH}, \zeta$-potential, and the relative viscosities of dispersions show that the relative viscosities decrease with decreasing absolute value of $\zeta$-potential, which further reveals the effect of electrical repulsion forces on the structure of aggregates.

This work was supported by the Research Foundation of Science and Technology Department of Zhejiang Province, China (2009C21023) and the China Postdoctoral Science Foundation (20080441248).

1 Eastman J A, Choi S U S, Li S, et al. Anomalously increased effective thermal conductivities of ethylene glycol-based nanofluids containing copper nanoparticles. Appl Phys Lett, 2001, 78: 718-720

2 Marquis F D S, Chibante L P F. Improving the heat transfer of nanofluids and nanolubricants with carbon nanotubes. JOM, 2005, 57: 32-44

3 Jin S, Ye K. Nanoparticle-mediated drug delivery and gene therapy. Biotechnol Prog, 2007, 23: 32-41

4 Yamanaka J, Ise N, Miyoshi H, et al. Experimental examination of the Booth theory on the first-order electroviscous effect in ionic colloidal dispersions. Phys Rev E, 1995, 51: 1276-1281

5 Wang $\mathrm{X}, \mathrm{Xu} \mathrm{X}$, Choi S U S. Thermal conductivity of nanoparticle-fluid mixture. J Thermophys Heat Trans, 1999, 13: 474-480

6 Rubio-Hernandez F J, Ayucar-Rubio M F, Velazquez-Navarro J F, et al. Intrinsic viscosity of $\mathrm{SiO}_{2}, \mathrm{Al}_{2} \mathrm{O}_{3}$ and $\mathrm{TiO}_{2}$ aqueous suspensions. $\mathrm{J}$ Colloid Interface Sci, 2006, 298: 967-972

7 Putra N, Roetzel W, Das S K. Natural convection of nano-fluids. Heat Mass Trans, 2003, 39: 775-784

$8 \mathrm{Yu} \mathrm{W}$, Xie $\mathrm{H}$, Chen L, et al. Investigation of thermal conductivity and viscosity of ethylene glycol based $\mathrm{ZnO}$ nanofluid. Thermochimica Acta, 2009, 491: 92-96

9 Lee J, Hwang K, Janga S P, et al. Effective viscosities and thermal conductivities of aqueous nanofluids containing low volume concentrations of $\mathrm{Al}_{2} \mathrm{O}_{3}$ nanoparticles. Int $\mathrm{J}$ Heat Mass Transfer, 2008, 51: 2651-2656

10 Prasher R, Song D, Wang J, et al. Measurements of nanofluid viscosity and its implications for thermal applications. Appl Phys Lett, 2006 89: 133108

11 Prasher R, Phelan P E, Bhattacharya P. Effect of aggregation kinetics on the thermal conductivity of nanoscale colloidal solutions (nanofluid). Nano Lett, 2006, 6: 1529-1534

12 Rooij R D, Potanin A A, Ende D, et al. Steady shear viscosity of weakly aggregating polystyrene latex dispersions. J Chem Phys, 1993, 
99: 9213-9223

13 Jiang W, Ding G, Peng H, et al. Modeling of nanoparticles' aggregation and sedimentation in nanofluid. Curr Appl Phys, 2010, 10: 934-941

14 Wang B, Zhou L, Peng X. A fractal model for predicting the effective thermal conductivity of liquid with suspension of nanoparticles. Int $\mathbf{J}$ Heat Mass Transfer, 2003, 46: 2665-2672

15 Waite T D, Cleaver J K, Beattie J K. Aggregation kinetics and fractal structure of $\gamma$-Alumina assemblages. J Colloid Interface Sci, 2001, 241: 333-339

16 Simha R. The influence of brownian movement on the viscosity of solutions. J Phys Chem, 1940, 44: 25-34

17 Sun Y, Li X, Duzgunesv N, et al. The shape parameter of liposomes and DNA-lipid complexes determined by viscometry utilizing small sample volumes. Biophys J, 2003, 85: 1223-1232
18 Berber S, Kwon Y, Tomanek D. Unusually high thermal conductivity of carbon nanotubes. Phys Rev Lett, 2000, 84: 4613-4616

19 Krieger I M. Rheology of monodisperse lattices. Adv Colloid Interface Sci, 1972, 3: 111-136

20 Einstein A. Eine neue Bestimmung der Molekiil-dimensionen. Ann Phys, 1911, 34: 591-592

21 Kok C M, Rudin A. Relationship between the hydrodynamic radius and the radius of gyration of a polymer in solution. Makromol Chem Rapid Commun, 1981, 2: 655-659

22 Keblinski P, Phillpot S R, Choi S U S, et al. Mechanisms of heat flow in suspensions of nano-sized particles (nanofluids). Int J Heat Mass Transfer, 2002, 45: 855-863

23 Chen C, Huang C, Tseng W, et al. Dispersion and rheology of surfactant-mediated silver nanoparticle suspensions. Appl Surf Sci, 2010, 257: $650-655$

Open Access This article is distributed under the terms of the Creative Commons Attribution License which permits any use, distribution, and reproduction in any medium, provided the original author(s) and source are credited. 